\title{
PUBLICATION BIAS IN CLINICAL RESEARCH SPONSORED BY PHARMACEUTICAL INDUSTRY
}

\author{
Albena Kerekovska ${ }^{1}$, Bistra Galunska ${ }^{2}$ \\ ${ }^{1}$ Faculty of Public Health, Department of Social Medicine and Healthcare Organisation, \\ ${ }^{2}$ Faculty of Pharmacy, Department of Pharmaceutical Technologies, \\ Medical University of Varna
}

\begin{abstract}
An increasing number of clinical trials are funded by the pharmaceutical industry, and the industry has strong commercial interests in research publications that present their products positively and enhance their sales. As main sponsor of clinical trials, the pharmaceutical industry has gained unprecedented control over the evaluation of its own products and communication of research results. There is mounting evidence that industry-sponsored trials are biased in favor of the sponsor's products.

Various potential ways have been described in which pharmaceutical concerns exert influence on the outcome of a study and its communication, however, publication bias proved to be a major cause for bias in industry-funded trials. Pharmaceutical industry and its sponsored investigators selectively report favorable outcomes, fail to publish whole studies with unfavorable results, or publish studies with favorable results multiple times. Such manipulation of research communication might greatly jeopardize the scientific basis for good clinical practice.

This article explores the aspects of clinical trial performance that can be affected by pharmaceutical company sponsorship, particularly emphasizing on results dissemination and publication. It is aimed at describing the relationship between pharmaceutical industry sponsorship and bias in research publication. By revealing the scope of the problem and its essence - it attempts to sensitize the scientific, professional and publishing societies and provoke actions to prevent and control the problem.
\end{abstract}

Keywords: clinical research, pharmaceutical sponsorship, publication bias

\section{INTRODUCTION}

Medical practice and drug treatment should rely on solid scientific evidence, and it is generally recognised that the standard basis for treatment

Address for correspondence: Albena Kerekovska, $M D, P h D$

Faculty of Public Health, Department of Social Medicine and Healthcare Organisation, Medical University of Varna 55 Marin Drinov Str.

9002 Varna, Bulgaria

e-mail:kerekovska_a@yahoo.com

Received: February 13, 2014

Accepted: April 3, 2014 guidelines is systematic reviews and meta-analyses of all randomised controlled trials (19). Scientific evidence can only be valid if the studies included in the reviews and meta-analyses represent the complete body of research and are not biased in such a way that conclusions misrepresent the effectiveness and safety of clinical interventions. However, as systematic reviews and meta-analyses are usually limited to publicly available data, several factors related to research dissemination and publication, can give rise to biased conclusions. These include selection of studies submitted or accepted for publication, inclusion of undetected duplicate publications, and selective reporting (19). 
Publication bias refers to the distortion of data in scientific journals resulting from the fact that studies with significant and positive results are more likely to be published than studies with unfavorable - negative or insignificant results. Clinical decision-making should be based on the totality of research evidence and not on a sample biased by selective publication only of studies that show favorable results (28).

Several actors (investigators, editors, and sponsors) affect whether and how scientific results reach the public domain (19). The role of the sponsor is especially influential for research dissemination - as it has access to all data on the investigated product and an obvious conflict of interest (5). In recent years sponsoring companies became very much involved in all aspects of research on their products. They often design the products, perform the analysis, write the paper, and decide whether, when and in what form to publish the results (16).

Clinical studies are increasingly funded by pharmaceutical companies. Pharmaceutical industry, as the main sponsor of clinical trials, has gained unprecedented control over the evaluation of its own products and communication of research results. In some multicenter trials, authors may not even have access to their own data. Indeed, as drug industry's influence over research has grown, so has the potential for bias in clinical trials. Drug companies now finance most clinical research on prescription drugs, and there is mounting evidence that they often skew the research and its reporting to make their drugs look better and safer (16). Such manipulation of research outcomes communication might greatly jeopardize the scientific basis for clinical practice.

This paper explores the aspects of clinical trial performance that can be affected by sponsorship of pharmaceutical companies, particularly emphasizing on results dissemination and publication. The aim is to describe the relationship between pharmaceutical industry sponsorship and bias in research publication. By revealing the scope of the problem and its essence - it attempts to sensitize the scientific, professional and publishing societies and provoke actions to prevent and control the problem.
An electronic literature search was conducted in Medline, Web of Science, Cochrane Library and other database web sources for the last 25 years.

\section{ESSENCE AND IMPACT OF PUB- LICATION BIAS IN CLINICAL RESEARCH}

Publication bias occurs when the publication of research results selectively depends on their nature and direction (28). The results of published studies will then be systematically different from those of unpublished studies. The fact that studies with positive or favourable results are more likely to be published than those with negative or unfavourable results has been widely confirmed $(6,18,19,30)$. Studies with positive or favourable results have been associated with other factors that could bias scientific evidence, such as: faster publication, publication in higher impact factor journals, a greater number of publications (including covert duplicate publications, more frequent citation, and more likely publication in English (18).

A variation of publication bias is outcome-reporting bias, which is the selective reporting of some outcomes but not others, depending on their nature and direction. Outcomes are not only selectively reported, but negative results are reported in a positive manner and conclusions are often not supported by results data (18). The existence of outcome-reporting bias in clinical research has also been confirmed by high-quality empirical studies $(18,28)$.

Publication bias is known to be a major problem in the medical literature (7). It has the potential to reduce the quality and safety of health care outcomes. As professional medical bodies construct evidence-based guidelines on the basis of published trial results, publication bias can compromise their recommendations. This contributes to excessive prescription of expensive new drugs whose efficacy is overestimated and risks underestimated. Moreover, because the evidence is distorted, patients do not receive adequate treatment and information (24).

Publication bias has a considerable impact in studies funded by pharmaceutical companies. By concealing the unfavourable evidence about efficacy and safety, pharmaceutical companies deceive physicians, their patients and, perhaps, shareholders $(8,9)$. 
Albena Kerekovska, Bistra Galunska

Publication bias can prevent data of potential importance from reaching practicing health care providers. Such concealment represents a serious abuse of the trust freely offered to study investigators by research subjects. Unpublished studies, when they are unnecessarily repeated, also waste resources.

As non-publication of research and its unfavorable results can distort scientific and medical knowledge, it is largely impossible to reliably assess the benefit and harm of medical drugs on the basis of published trials. This will have repercussions for the reliability of meta-analyses, guidelines and patient information leaflets (2). A serious consequence may be undesired treatment errors. Indeed, recent controversies in varied medical fields have highlighted the importance of having access to such unpublished data. Previously underappreciated unpublished side effect data have led to removal of certain agents from the pharmaceutical market (7). Marketing of other agents, for unproven treatment indications, also has led to controversies and even to malpractice lawsuits. For instance, a review of antidepressant randomized controlled trial (RCTs) in unipolar depression in the Food and Drug Administration (FDA) database found that almost all negative studies were unpublished, leading to a false impression in the published literature that $93 \%$ of antidepressant RCTs had positive results; when unpublished studies were included, $51 \%$ of all RCTs were positive and $49 \%$ were negative (7). Thus, unpublished studies raise questions of concerns regarding both underreported risks and underreported limitations in efficacy.

With its negative impacts on clinical practice and ethical breaches - publication bias is generally accepted as serious scientific malpractice.

\section{INDUSTRY-INDUCED BIAS IN \\ CLINICAL TRIALS}

Most clinical trials are sponsored by pharmaceutical companies, and commercial interests may determine the dissemination profile of a study. There has been an increasing number of studies suggesting that industry sponsored trials are biased in favor of the sponsor's products (14).

Many systematic reviews have demonstrated that pharmaceutical industry sponsorship of drug and medical device studies is associated with findings that are favorable to the sponsor's product
$(2,3,9,14,25,27)$. Industry sponsored drug and device studies more often have favorable efficacy results, harms results and overall conclusions, compared with non-industry sponsored drug and device studies. Bekelman and colleagues found that industry sponsorship was very strongly associated with proindustry conclusions (3). These findings were supported by Ridker and Torres, who carefully analyzed the randomized cardiovascular trials published in JAMA, The Lancet, and The New England Journal of Medicine. They found that the proportion of studies favoring newer treatments over the standard of care was significantly higher in trials funded by for-profit organizations compared with trials funded by notfor-profit organizations (21).

Reviews of the impact of financial conflicts on biomedical research revealed that studies financed by industry always found outcomes favourable to the sponsoring company (14). It was demonstrated that industry-funded studies tend to emphasize favourable beneficial effects of the sponsor's product, and to underestimate the adverse effects and patients' risks. The nonpublication of negative outcomes in RCTs has been especially reported to occur in pharmaceutical industry-funded studies, as opposed to studies funded by other sources (7).

It has been shown that more than half of the studies that are conducted as part of the drug approval process will remain unpublished and these are trials funded by for-profit companies failing to demonstrate a positive outcome for the company's product. In addition, multiple publications of the same results, the selective use of data and the withholding of data relating to adverse drug reactions were widely demonstrated (14). Such findings apply across a wide range of disease states, drugs, and drug classes, and regardless of the type of research being assessed - pharmacoeconomic studies, clinical trials, or metaanalyses of clinical trials (3). The evidence suggests that there is some kind of systematic bias to the outcome of published research funded by the pharmaceutical industry (14).

Systematic investigations have been carried out aiming to spotlight the different stages and aspects of drug trials that are influenced by funding from the pharmaceutical industry. Various potential ways have been described in which pharmaceutical con- 
cerns exert influence on the outcome of a study and its communication, including the design of the study protocol, the conduct of the drug trial, the analysis of data, selective reporting of favorable results, and spin in reporting conclusions $(23,26)$.

Numerous examples have been found of bias induced by sponsorship from pharmaceutical industry in the areas of: research question, choice of dose and comparator agents, control over trial design and changes in protocols, early termination of clinical trials, reporting to regulatory authorities, reinterpretation of data, restrictions on publication rights, use of fake journals, journal supplements and symposia, ghostwriting, publication and reporting of results and outcomes. On the other hand, the release of internal industry documents as a result of settlement agreements resulting from litigation against drug companies has revealed examples of industry manipulation of the conduct and publication of studies (15).

There has been evidence that pharmaceutical companies influenced study protocols in a way that was favorable to them. Execution of the study according to plan and objective depiction of the results can also be influenced, e.g., by contractual stipulations that grant the pharmaceutical company access to the trial data or give it the power to prevent the publication of results (24).

Studies have empirically demonstrated that industry preferentially supports trial designs that favor positive results, such as: the use of placebo as the comparison therapy in controlled trials, active comparators in inferior doses, or inappropriate administration of the drugs $(1,12)$. The frequent use of placebo controls in clinical trials has been often attributed to Food and Drug Administration regulations; however, the FDA does not require the use of placebo and is supportive of trials that incorporate active controls. Use of active controls does not eliminate the potential for bias; for instance, evidence from NSAID and fluconazole RCTs has revealed that inappropriate administration and dosing disparities decrease the effectiveness of active controls (3). In the study by Rochon et al., in most cases in which the doses of the study and comparator drugs were not equivalent, the drug given at the higher dose was that of the supporting manufacturer (22). Higher doses may bias the re- sults in favour of effectiveness of the manufacturer's product.

Pharmaceutical trials have been accused elsewhere in the literature of using design modifications to ascertain lower adverse effects. Such methods may potentially include using lower doses of the intervention and higher doses for the controls, monitoring for adverse effects using open-ended or nonspecific questions, and the choice of inappropriate comparators. Industry sponsored studies may be biased in the coding of events and their data analysis (15) in a way that underestimate the undesired adverse effects of the investigated company's product. Systematic reviews also indicate that the funding source may impact on the nature of the authors' interpretation and conclusions regarding the safety profile (9).

\section{PUBLICATION BIAS IN INDUSTRY- SPONSORED RESEARCH}

Selective publication and outcome-reporting (publication bias) prove to be a major cause for bias in industry funded trials. Pharmaceutical industry and its sponsored investigators may selectively report favorable outcomes, fail to publish whole studies with unfavorable results, or publish studies with favorable results multiple times (15).

Many cases have been described in which the publication of studies with negative results has been suppressed by pharmaceutical companies. In the past few years, manufacturers have attempted to prevent studies which are unfavourable to their products from being published in several high profile cases $(6,18,29)$. The withholding of negative and statistically non-significant findings can result in publication bias, leading to a distorted perception of the therapeutic value of the drug concerned (24).

Further studies reveal evidence of other problems including incomplete trial registration, constraints on publishing rights, withheld knowledge of adverse drug reactions, and the use of ghostwriters who were supplied by the pharmaceutical companies. The presentation of results can be manipulated by ghostwriters and guest authors, where a wellknown opinion former is invited to be a guest author in order to underline the importance of the study results. 
Consistent evidence also demonstrates that industry ties are associated with both publication delays and data withholding. These restrictions, often contractual in nature, serve to compound bias in biomedical research. In addition, industry financing is also connected with multiple reporting of studies with positive outcomes, further compounding publication bias and potentially swaying review articles toward more positive results (18).

Massie and colleagues raised another possible source of publication bias. They showed that research funded by the industry appears more often in symposiums (17). Studies in symposiums are known to lack peer review and to favour the sponsor's product, therefore the absence of peer review may result in an overly favourable interpretation of the results of a trial. Rochon and colleagues noted that claims of superiority for the sponsor's product claimed in symposia presentations, were often not supported by the data (15).

\section{CONFLICT OF INTEREST IN IN- DUSTRY-SPONSORED RESEARCH}

Pharmaceutical companies fund the vast majority of the clinical research that is undertaken on medications but face a conflict of interest between producing good science and results that will enhance the sales of their products (13). This entails an obvious contradiction, as on one side, drugs as a technology are used to cure diseases and relieve symptoms; on the other, they are produced mainly by corporations with a firm footing in the economic market and are subject to the market's laws and driven by the pursuit of profit.

The pharmaceutical and medical device industries have strong interests in scientific publications that present their products positively, as publications are the basis of regulatory, purchasing, and medical decisions (4). Results and conclusions that are unfavorable to the sponsor (i.e. studies that find an expensive drug similarly or less effective or more harmful than drugs used to treat the same condition) can pose considerable financial risks to the companies. Therefore, drug companies may be particularly unwilling to publish sponsored trials with unfavourable results (15). Companies may also try to prevent the publication of studies conducted by others when the findings will undermine their commercial inter- ests. These interests can influence the design, conduct and publication of studies in ways that make the sponsor's product appear better than the comparator product (16). The pharmaceutical industry has less to do with health than with business, investments, stock prices, and financial gain. This contradiction produces devastating effects that have become progressively more visible to health professionals and researchers, triggering vigorous challenges (16).

\section{PREVENTION OF PUBLICATION BIAS IN INDUSTRY-SPONSORED RESEARCH}

An effective approach for prevention of publication bias induced by industry should consider the financial conflicts of interest in clinical research. The safety of patients must remain the paramount concern, bias in the research process must be minimized, and appropriate incentives for research innovation must be preserved. For the purpose, bias preventive measures must take into account the industrialization of clinical research and the conflict of interests involved in industry-sponsored trials.

Both medical associations and research journal editors have become concerned with conflicts of interest in the conduct of clinical research. Editors and academics have criticised companies for selective reporting of clinical trials, duplicate publication and various other forms of manipulation of research outcome communication. Guideline documents have been developed to address these issues and increase the credibility of studies paid for by drug companies.

Leading medical journals established more rigorous criteria for the acceptance of research sponsored by industry (14). The ICMJE Uniform requirements revision (11) calls for full disclosure of the sponsor's role in research, as well as assurance that the investigators are independent of the sponsor, are fully accountable for the design and conduct of the trial, have independent access to all trial data and control all editorial and publication decisions. The CONSORT (Consolidated standards of reporting trials) Statement was endorsed by prominent medical journals and leading editorial organizations (14). Pharmaceutical organizations implemented recommendations that are intended to ensure comprehensive publication of research findings, whether positive or negative. In 2002, the Pharmaceutical Re- 
Publication bias in clinical research sponsored by pharmaceutical industry

search and Manufacturers Association (PhRMA) published its "Principles on the Conduct of Clinical Trials and Communication of Clinical Trial Results" (20). Similarly, Good Publication Practice (GPP) for pharmaceutical companies was also set up aimed at improving the behaviour of drug companies while reporting drug trials (10).

Besides these initiatives on the part of medical organizations, leading journals and the pharmaceutical industry - negative results of industry sponsored research are still not published in timely fashion and control mechanisms fail. The findings of recent studies suggest that investigators and sponsors undertaking human participant research do not adhere to the endorsed standards of accountability, access to data and control of publication (10).

Mandatory prospective registration of clinical trials and full study results after study completion, as well as public access to study data via results databases need to be introduced on a worldwide level (18). This would help fulfil ethical obligations towards patients by enabling proactive publication and independent reviews of clinical trial data, and ensure a basis for fully informed decision making in the health care system. Otherwise, clinical decision making based on the „best evidence“ will remain an illusion.

\section{CONCLUSIONS}

An increasing number of clinical trials are funded by the pharmaceutical industry, and the industry has strong interests in scientific publications that present their products positively. Strong and consistent evidence shows that industry-sponsored research tends to draw pro-industry biased conclusions. Publication bias is proved to be a major cause for bias in industry funded trials. Published drug trials that are financed by pharmaceutical companies may often present a distorted picture, which seriously impacts on validity of scientific evidence - a basis for clinical decisions. Therefore rigorous coherent measures must be taken by all - scientific and medical organizations, ethical committees, regulatory bodies, journal editors and the industry itself - to ensure that commercial pharmaceutical interests do not undermine the knowledge of scientifically correct study planning, study execution, and publication.

\section{REFERENCES}

1. Als-Nielsen B, Chen W, Gluud C, Kjaergard LL. Association of funding and conclusions in randomized drug trials: a reflection of treatment effect or adverse events? JAMA. 2003;290(7):921-8.

2. Becker-Brüser W. Research in the pharmaceutical industry cannot be objective. Z Evid Fortbild Qual Gesundhwes. 2010;104(3):183-9.

3. Bekelman J, Li Y, Gross C. Scope and impact of financial conflicts of interest in biomedical research: A systematic review. JAMA. 2003;289(4):454-65.

4. Bero LA, Rennie D. Influences on the quality of published drug studies. Int J Technol Assess Health Care. 1996;12(2):209-37.

5. Davidoff F, DeAngelis C, Drazen J, Hoet J, Højgaard L, Horton R, et al. Sponsorship, authorship, and accountability (commentary). Lancet. 2001;358:854-6.

6. Easterbrook PJ, Berlin JA, Gopalan R, Matthews DR. Publication bias in clinical research. Lancet. 1991;337:867-72.

7. Ghaemi SN, Shirzadi AA, Filkowski M. Publication bias and the pharmaceutical industry: The case of Lamotrigine in bipolar disorder. Medscape J Med. 2008;10(9):211.

8. Goldacre B. Bad Pharma: How drug companies mislead doctors and harm patients. London: Fourth Estate; 2012.

9. Golder $\mathrm{S}$, Loke $\mathrm{Y}$. Is there evidence for biased reporting of published adverse effects data in pharmaceutical industry-funded studies? $\mathrm{Br} \mathrm{J}$ Clin Pharmacol. 2008;66(6):767-73.

10. Graf C, Battisti W, Bridges D, Winkler V, et al. Good publication practice for communicating company sponsored medical research: the GPP2 guidelines. BMJ. 2009;339:b4330.

11. ICMJE. Recommendations for the conduct, reporting, editing and publication of scholarly work in medical journals. [Cited $2014 \mathrm{Feb}$ 1]. Available from: http://www.icmje.org/new_recommendations.html

12. Lathyris DN, Patsopoulos NA, Salanti G, Ioannidis JP. Industry sponsorship and selection of comparators in randomized clinical trials. European Journal of Clinical Investigation. 2010;40(2):172-82.

13. Lexchin J. Sponsorship bias in clinical research. Int J Risk Saf Med. 2012; 24(4): 233-42. 
14. Lexchin J, Bero L, Djulbegovic B, Clark O. Pharmaceutical industry sponsorship and research outcome and quality: systematic review. BMJ. 2003; 326(7400):1167.

15. Lundh A, Sismondo S, Lexchin J, Bero L. Industry sponsorship and research outcome (Review). The Cochrane Collaboration, JohnWiley \& Sons; 2013.

16. Marcia A. Industry-Sponsored clinical research: $A$ broken System. JAMA. 2008;300(9):1069-71.

17. Massie BM, Rothenberg D. Publication of sponsored symposiums in medical journals. N Engl J Med. 1993;328:1196-7.

18. McGauran N, Wieseler B, Kreis J, Schüler YB, Kölsch H, Kaiser T. Reporting bias in medical research - a narrative review. BioMed Central Trials. 2010;11:37.

19. Melander H, Ahlqvist-Rastad J, Meijer G, Beermann B. Evidence b(i)ased medicine: selective reporting from studies sponsored by pharmaceutical industry; review of studies in new drug applications. BMJ. 2003;326:1171-3.

20. PhRMA. Principles on the conduct of clinical trials and communication of clinical trial results. 2011 Jul; 1-44. Available from: http:// www.phrma.org/publications/policy_papers/ phrma_clinical_trial_registry_

21. Ridker PM, Torres J. Reported outcomes in major cardiovascular clinical trials funded by forprofit and not-for-profit organizations: 2000-2005. JAMA. 2006;295(19):2270-4.

22. Rochon PA, Gurwitz JH, Simms RW, Fortin PR, Felson DT, Minaker KL, et al. A study of manufacturer-supported trials of nonsteroidal anti-inflammatory drugs in the treatment of arthritis. Arch Intern Med. 1994;154:157-63.
23. Schott G, Pachl H, Ludwig W. The relation between publication bias and clinical trials funding. Z Evid Fortbild Qual Gesundhwes. 2010;104(4):314-22.

24. Schott G, Pachl H, Limbach U, Gundert-Remy U, Lieb K, Ludwig W. The financing of drug trials by pharmaceutical companies and its consequences: Part 2. A qualitative, systematic review of the literature on possible influences on authorship, access to trial data, and trial registration and publication. Dtsch Arztebl Int. 2010;107(17):295-301.

25. Schott G, Pachl H, Limbach U, Gundert-Remy U, Ludwig W, Lieb K. The financing of drug trials by pharmaceutical companies and its consequences. Part 1: A qualitative, systematic review of the literature on possible influences on the findings, protocols, and quality of drug trials. Dtsch Arztebl Int. 2010;107(16):279-85.

26. Sismondo S. How pharmaceutical industry funding affects trial outcomes: Causal structures and responses. Social Science \& Medicine. 2008 Feb 7. doi: 10.1016/j.socscimed.2008.01.010

27. Sismondo S. Pharmaceutical company funding and its consequences: a qualitative systematic review. Contemporay Clinical Trials. 2008;29(2):109-13.

28. Song F, Eastwood AJ, Gilbody S, Duley L, Sutton AJ. Publication and related biases. Health Technology Assessment. 2000;4(10):1-105.

29. Song F, Parekh S, Hooper L, Loke YK, Ryder J, Sutton AJ, et al. Dissemination and publication of research findings: an updated review of related biases. Health Technol Assess. 2010 Feb;14(8):iii, ix-xi, 1-193.

30. Stern JM, Simes RJ. Publication bias: evidence of delayed publication in a cohort study of clinical research projects. BMJ. 1997;315:640-5. 